\title{
Synthesis of novel twisted heterocyclic analogues of $s$-indacenes
}

\author{
Krzysztof S. Danel, ${ }^{a, *}$ Tomasz Uchacz, ${ }^{b}$ and Marcin Karelus ${ }^{b}$ \\ ${ }^{a}$ Department of Chemistry, University of Agriculture, Balicka 122, 30-149 Kraków, Poland \\ ${ }^{b}$ Department of Chemistry, Jagiellonian University, Ingardena 3, 30-060 Kraków, Poland \\ E-mail: $\underline{\text { rdanelk@cyf-kr.edu.pl }}$
}

\begin{abstract}
A series of novel compounds as candidates for OLED applications were synthesized by cyclization reactions of neighbouring rings in dipyrazolo[3,4-b:4',3'-e]pyridines 8-12. The desymmetrization of these molecules leading to $\mathbf{1 4}$ can be performed using both $\mathrm{KOH} /$ isoquinoline or palladium catalysts. The resulting structures contain a helical arrangement of four different rings.
\end{abstract}

Keywords: Aza-s-indacenes, helicenes, synthetic methods, organic diodes

\section{Introduction}

In our previous reports we successfuly used $\mathrm{KOH} /$ (iso)quinoline system for the production of 6aryl-6H-5,6,7-triazadibenzo[f,h]naphtho[3,2,1-cd]azulenes $\mathbf{3}$ originating from 4-(2-halophenyl)$1 H$-pyrazolo[3,4-b]quinolines 2 (Figure 1). ${ }^{1}$

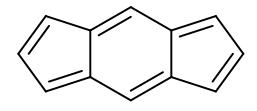

1
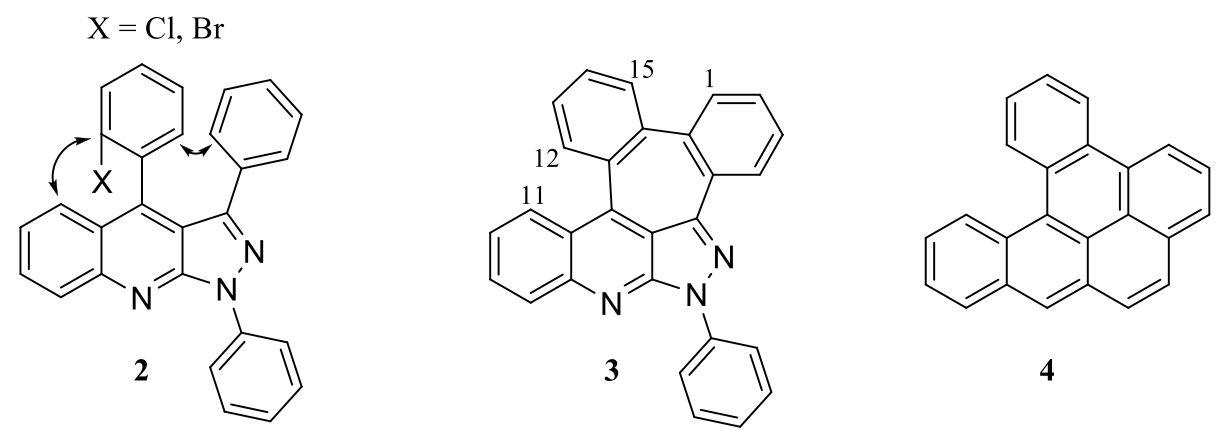

Figure 1. $1 s$-indacene; 2 4-(2-halophenyl)-1,3-diphenyl-1H-pyrazolo[3,4-b]quinoline; 3 6phenyl-6H-5,6,7-triazadibenzo[f,h]naphtho[3,2,1-cd]azulene, with atomic numbering; 4 dibenzo[ $[a, l]$ pyrene. 
These new dyes are characterized by the presence of a seven-membered ring adjoining aromatic moieties and may be of interest for luminescent or electroluminescent applications. Similar arrangements of rings can be found in a variety of biologically active compounds, ${ }^{2 a}$ novel nucleosides, ${ }^{2 b}$ carbon nanotubes as so called Stone-Wales defects, ${ }^{3}$ or in recently synthesized dimeric triazadibenzo[ $c d, g]$ azulenes. ${ }^{4}$ These angularly arranged rings in $\mathbf{3}$ impose helicity on the molecules and should prevent to some extent their aggregation in the bulk thus extending the lifetime of a potential organic light-emitting diode (OLED). This distortion, reminiscent of that encountered in either dibenzo[a,l]pyrene ${ }^{5} \mathrm{DB}[a, l] \mathrm{P} 4$, or benzo[c]phenanthrene ([4]helicene), ${ }^{6}$ is the result of strongly interacting protons at the positions 1,15 and 11,12 in $\mathbf{3}$, respectively. Recently, 3,12-dimethoxy-7,8-dicyano[5]helicene was evaluated as a novel emissive material for organic light-emitting diodes. ${ }^{7}$ In this communication, we report a new series of dyes, modified aza $s$-indacenes 14, derived from 4-(2-halophenyl)-1,3,5,7-tetraaryl-1,7-dihydrodipyrazolo[3,4$\left.b ; 4^{\prime}, 3^{\prime}-e\right]$ pyridines 8-12. Dipyrazolopyridine derivatives have recently been extensively tested as promising blue emitters in OLEDs. ${ }^{8}$ The emission maxima of newly designed dyes are shifted to a longer wavelength.

\section{Results and Discussion}

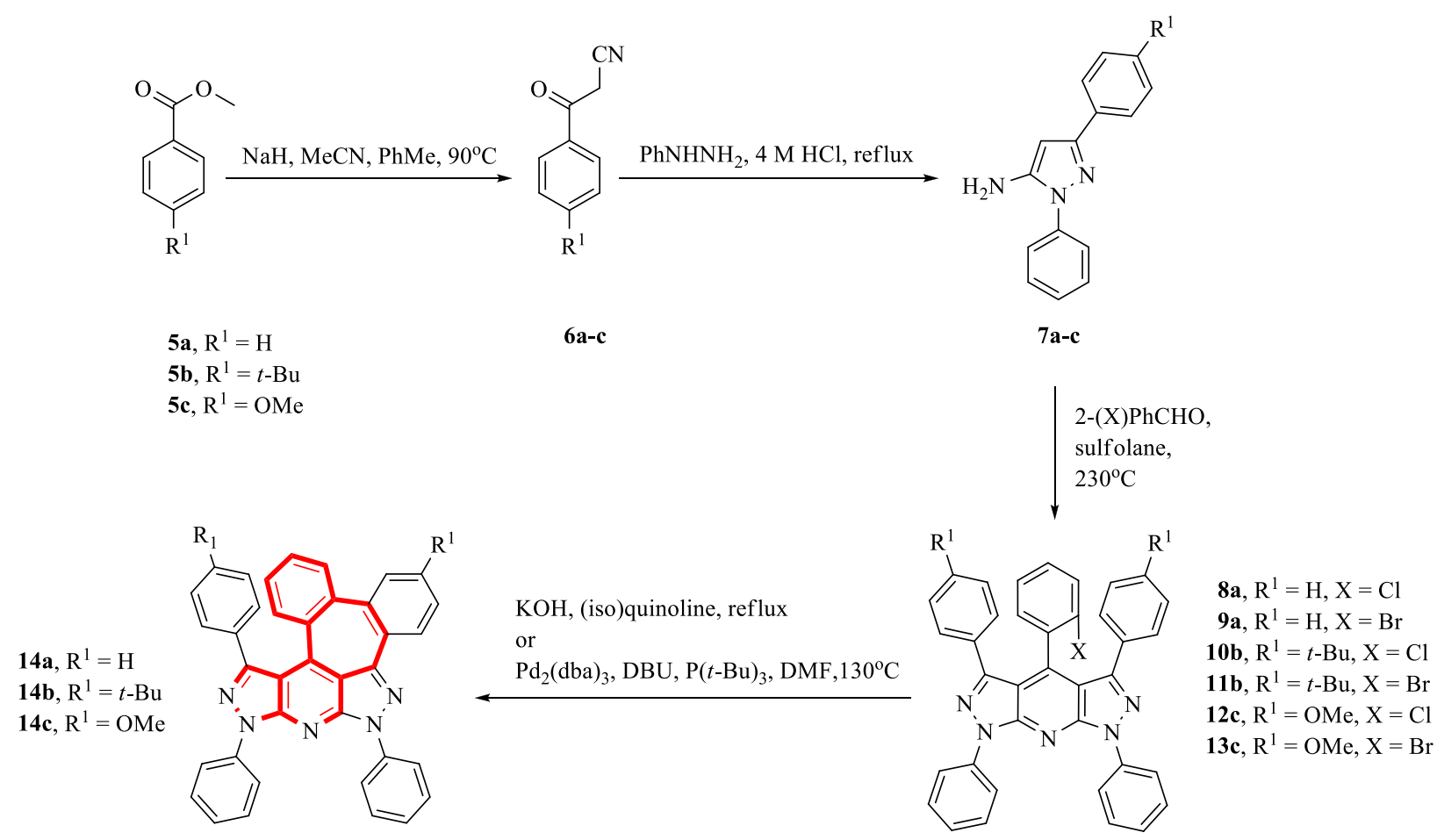

\section{Scheme 1}


Pyrazolo[3,4-b]quinoline 2 (PQ) has two possible sites for cyclization (Figure 1). In the case of (iso)quinoline/KOH this preference is meaningless: always a seven-membered ring is formed. ${ }^{1}$ Palladium-assisted ring closure is not so obvious: in some cases there are negligible amounts of seven-membered isomers formed apart from the main five-membered ring products. ${ }^{1 a}$ This holds true if the substituent at the 6-position of the PQ skeleton is not bulky enough, or secondary amine. These precautions were kept in mind when we turned our attention to 1,3,4,5,7pentaphenyl-1,7-dihydrodipyrazolo[3,4-b:4',3'-e]pyridines. These interesting aza analogues of $s$ indacene $\mathbf{1}$ have a plane of symmetry, thus they are devoid of the aforementioned obstacles. Therefore, it was tempting to use our procedure to create the novel annulated systems 14, 1,3,5triphenyl-2,3,4,5,6-pentaazadibenzo[4,5:6,7]cyclohepta[1,2,3-cd]s-indacenes, abbreviated as TPPACI. The aforementioned bispyrazolo[3,4-b:4',3'-e]pyridines can be obtained in several steps $(4,3$ or 2$)$ starting from commercially available aromatic carboxylic acids, methyl benzoates 5 or aroylacetonitriles 6 (Scheme 1). Finally, these by treatment with phenylhydrazine in boiling $4 \mathrm{M}$ hydrochloric acid, gave aminopyrazoles 7 . The reaction of these with 2-halobenzaldehydes in sulfolane delivered precursors 8-13. However the conversion from $\mathbf{7}$ to 8-13 did not always go in the same way. Condensation of $\mathbf{7 c}\left(\mathrm{R}^{1}=\mathrm{OMe}\right)$ with 2-bromobenzaldehyde yielded the expected product 13c only in a trace amount (TLC). After replacement of the aldehyde with its chloro analogue we obtained 12c in $21 \%$ yield after column purification. Reaction of pyrazole $7 \mathbf{a}$ with 2-halobenzaldehydes delivered 8a and 9a, in 35\% and 32\% yields respectively, which did not require chromatographic isolation. The preparation of both tert-butylated analogues of bispyrazolopiridines 10-11b did not encounter any problems either, although chromatographic purification was performed to afford the pure products. The rationale to prepare bromo derivatives was to attempt a ring closure under palladium catalysis to avoid drastic conditions. First to afford TPPACI we cyclized ( $\mathrm{KOH}$, isoquinoline, method A) our intermediates 8-12 as in previous reports. ${ }^{1}$ We observed that bromo-analogues reacted faster (1 hour). For chloro derivatives, 2 hours were required to complete the conversion. Generally, reaction times were shorter, because the 2-halophenyl moiety has two neighboring phenyls to be attacked, compared to one in 4-(2-halophenyl)- $1 H$-pyrazolo[3,4-b]quinolines $2 .{ }^{1}$ Palladium-assisted cyclization performed (method B) on 9a and 11b delivered the targets after 5 hours in good yields. The progress of reaction could be easily monitored by TLC: intensely blue emitting spots of $\mathbf{9 a}$ and $11 \mathrm{~b}$ were converted to less polar TPPACI of yellow emission. Compound $\mathbf{1 4 b}$ was more soluble than 14a and 14c. It also had a high melting point (Table 1). 


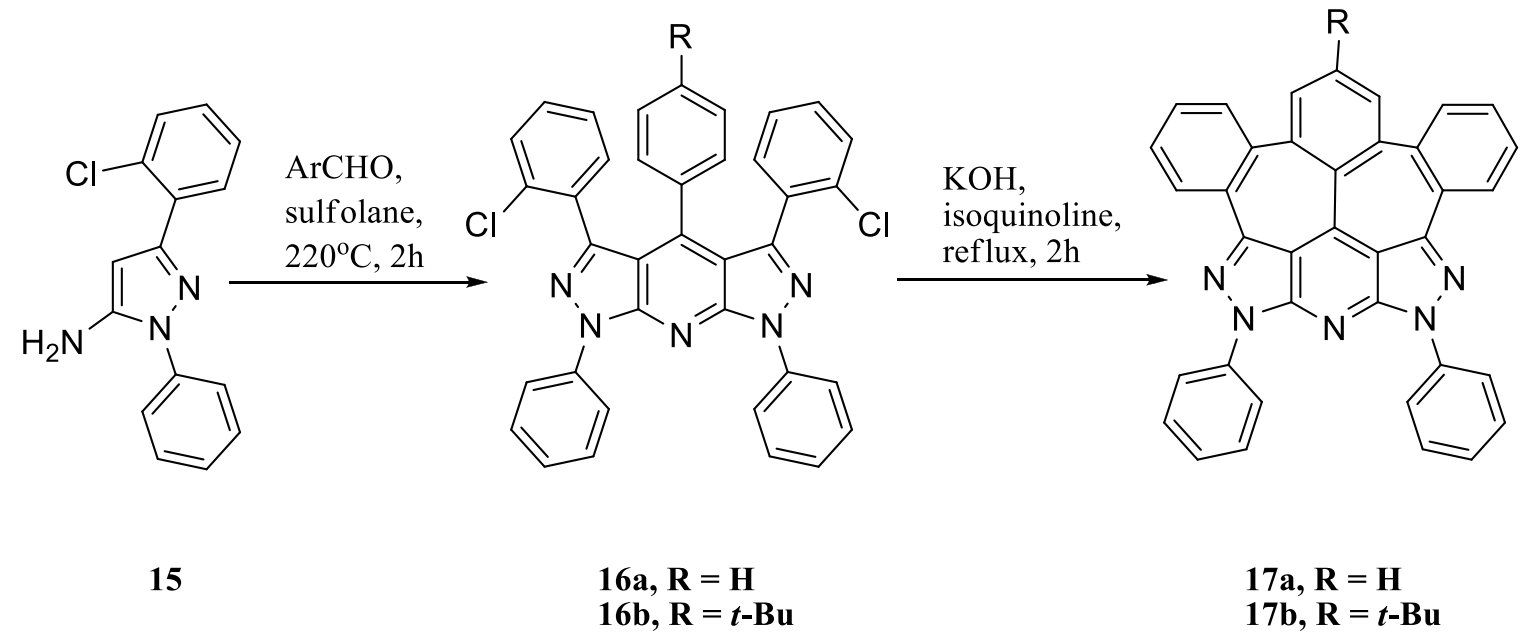

\section{Scheme 2}

The above results encouraged us to try a double cyclization to obtain $\mathbf{1 7 a}$ (Scheme 2). The first attempt was not satisfactory, because the target compound was extremely insoluble in all common organic solvents $\left(\mathrm{mp}>350{ }^{\circ} \mathrm{C}\right)$. It could only be dissolved in boiling DMAC, DMSO or 1-methyl-2-pyrrolidinone. Taking into account the solubility studies performed on TPPACI we introduced a tertiary butyl to make the compound $\mathbf{1 7 b}$ amenable for further treatment. Indeed, this moiety improved the solubility substantially and we were able to record both ${ }^{1} \mathrm{H}$ and ${ }^{13} \mathrm{C}$ NMR spectra. It also had a high melting point $\left(339-341{ }^{\circ} \mathrm{C}\right)$. The absorption $\left(\lambda_{\text {abs }}\right)$ and luminescence $\left(\lambda_{\mathrm{fl}}\right)$ data of the annulated compounds are summarized in Table 1. One can observe a slight bathochromic shift in the peak maximum of absorbance in going from 14a to 14c, and a 'jump' of $70 \mathrm{~nm}$ for completely cyclized $\mathbf{1 7 b}$. A similar effect was also found for the emission peak maximum. The quantum yields decreased from 0.50 for $\mathbf{1 4 a}$ to 0.32 for $\mathbf{1 4 c}$. These data are in the range of already reported values. ${ }^{8 \mathrm{~d}}$ The smaller quantum yield of yellow-orange fluorescence $\mathbf{1 7 b}$ falls within the range of red emitting benzo[ $a]$ aceanthrylene derivatives. ${ }^{9}$

Table 1. Preparation of TPPACI and 17b from bispyrazolo[3,4- $b ; 4^{\prime}, 3$ '-e $]$ pyridines $\mathbf{8 - 1 2 , 1 6 b}$ and photophysical properties of investigated compounds in cyclohexane (see Experimental Section).

\begin{tabular}{|c|c|c|c|c|c|c|c|}
\hline \multirow{2}{*}{ Entry } & \multicolumn{2}{|c|}{ Method, Yield (\%) } & \multirow{2}{*}{ TPPACI } & \multirow{2}{*}{$\lambda_{\mathrm{abs}}[\mathrm{nm}]$} & \multirow{2}{*}{$\lambda_{\mathrm{fl}}[\mathrm{nm}]$} & \multirow{2}{*}{$\Phi_{\mathrm{fl}}$} & \multirow{2}{*}{$\operatorname{Mp}\left({ }^{\circ} \mathrm{C}\right)$} \\
\hline & A & B & & & & & \\
\hline $\mathbf{8 a}$ & 83 & - & \multirow[b]{2}{*}{$14 a$} & \multirow[b]{2}{*}{440} & \multirow[b]{2}{*}{520} & \multirow[b]{2}{*}{0.50} & \multirow[b]{2}{*}{$282-284$} \\
\hline $9 a$ & 99 & 92 & & & & & \\
\hline $10 b$ & 60 & - & \multirow[b]{2}{*}{$14 b$} & \multirow[b]{2}{*}{444} & \multirow[b]{2}{*}{525} & \multirow[b]{2}{*}{0.45} & \multirow[b]{2}{*}{$320-322$} \\
\hline $11 b$ & 51 & 74 & & & & & \\
\hline $12 \mathrm{c}$ & 85 & - & $14 c$ & 452 & 547 & 0.32 & $291-293$ \\
\hline $16 b$ & 81 & - & $17 b$ & $459,487,522$ & 576 & 0.08 & $339-341$ \\
\hline
\end{tabular}




\section{Conclusions}

In summary, a new and general synthesis of heterocyclic indacenes was developed using two methods: a classical approach based on $\mathrm{KOH} /$ isoquinoline and, using milder conditions, employing $\mathrm{Pd}_{2}(\mathrm{dba})_{3} / \mathrm{P}(t-\mathrm{Bu})_{3}$ catalytic systems. We also obtained a trisubstituted octacyclic azarene $\mathbf{1 7 b}$, which could possibly be used as a fluorescent core $^{10}$ to generate novel and interesting dendrimers. Further studies are in due course to expand the scope of the present method (palladium cyclization of chlorinated analogues of bispyrazolo[3,4- $\left.b ; 4^{\prime}, 3^{\prime}-e\right]$ pyridines), and to use the products for further conversion as well as for LED purposes.

\section{Experimental Section}

General. ${ }^{1} \mathrm{H}$ NMR and ${ }^{13} \mathrm{C}$ NMR spectra were recorded using a Mercury-Vx $300 \mathrm{MHz}$ Varian operating at 300 and $75 \mathrm{MHz}$, respectively, in $\mathrm{CDCl}_{3}$ with tetramethylsilane (TMS) as an internal standard. Elementary analyses were performed on a Perkin-Elmer $2400 \mathrm{CHN}$ analyzer. Melting points were measured on a Melt-Temp II instrument. The purity of the compounds obtained was checked by TLC. Methyl benzoates $\mathbf{5}$ were commercially available or were synthesized by heating the corresponding acids at reflux with $\mathrm{MeOH}$ in the presence of concentrated sulfuric acid. 3-Oxonitriles 6 were produced by reaction of the esters with acetonitrile in the presence of $60 \%$ suspension of sodium hydride in toluene. ${ }^{11}$ Aminopyrazoles $\mathbf{7}$ and $\mathbf{1 5}$ were prepared following the method described in the literature. ${ }^{12}$ Finally, bispyrazolopyridines 8-12 and 16a,b were obtained via reported methods. ${ }^{13}$ Absorption $\left(\lambda_{\mathrm{abs}}\right)$ and emission $\left(\lambda_{\mathrm{abs}}\right)$ measurements were carried out using a scanning spectrophotometer UV-VIS 2101 (Shimadzu) and a conventional fluorescence spectrometer supplied with the cooled photomultiplier EMI $9558 \mathrm{~B}$, respectively. Emission quantum yields $\left(\Phi_{\mathrm{fl}}\right)$ were measured using quinine sulfate in $0.05 \mathrm{M} \mathrm{H}_{2} \mathrm{SO}_{4}$ solution as an actinometer. Both the optical absorption and fluorescence spectra were measured at room temperature in cyclohexane with the molar concentration of the dyes being about $10^{-5} \mathrm{M}$ in each case.

4-(2-Chlorophenyl)-1,3,5,7-tetraphenyl-1,7-dihydrodipyrazolo[3,4-b;4',3'-e]pyridine $\quad(8 \mathrm{a})$. Yield $2.02 \mathrm{~g}(35 \%)$; light yellow solid; $\mathrm{mp}=251-253{ }^{\circ} \mathrm{C} ; \mathrm{R}_{\mathrm{f}}=0.55$ (toluene); ${ }^{1} \mathrm{H} \mathrm{NMR}\left(\mathrm{CDCl}_{3}\right.$, $300 \mathrm{MHz}) \delta 6.79(\mathrm{td}, 1 \mathrm{H}, J=7.5,1.2 \mathrm{~Hz}), 6.88-6.94(\mathrm{~m}, 2 \mathrm{H}), 6.99-7.05(\mathrm{~m}, 5 \mathrm{H}), 7.11-7.18(\mathrm{~m}$, $6 \mathrm{H}), 7.34(\mathrm{t}, 2 \mathrm{H}, J=7.5 \mathrm{~Hz}), 7.58(\mathrm{t}, 4 \mathrm{H}, J=7.5 \mathrm{~Hz}), 8.55(\mathrm{~d}, 4 \mathrm{H}, J=8.4 \mathrm{~Hz}) ;{ }^{13} \mathrm{C}$ NMR $\left(\mathrm{CDCl}_{3}, 75 \mathrm{MHz}\right) \delta 112.68,120.81,125.66,125.76,127.35,127.77,128.82,128.99,129.07$, 129.87, 131.19, 132.25, 133.04, 133.24, 138.24, 139.60, 147.82, 150.59; Anal. Calcd. for $\mathrm{C}_{37} \mathrm{H}_{24} \mathrm{ClN}_{5}$ : C, 77.41; H, 4.21; N,12.20; found: C, 77.21; H, 4.44; N, 12.11 .

4-(2-Bromophenyl)-1,3,5,7-tetraphenyl-1,7-dihydrodipyrazolo[3,4-b;4',3'-e]pyridine (9a). Yield $991 \mathrm{mg}$ (32\%); yellow solid; $\mathrm{mp}=242-243{ }^{\circ} \mathrm{C} ; \mathrm{R}_{\mathrm{f}}=0.34$ (toluene/PE, 1:1); ${ }^{1} \mathrm{H}$ NMR $\left(\mathrm{CDCl}_{3}, 300 \mathrm{MHz}\right) \delta 6.82-6.96(\mathrm{~m}, 3 \mathrm{H}), 7.00-7.05(\mathrm{~m}, 4 \mathrm{H}), 7.11-7.18(\mathrm{~m}, 7 \mathrm{H}), 7.34(\mathrm{t}, 2 \mathrm{H}, J=$ 
$7.5 \mathrm{~Hz}), 7.58(\mathrm{t}, 4 \mathrm{H}, J=7.5 \mathrm{~Hz}), 8.55(\mathrm{~d}, 4 \mathrm{H}, J=8.7 \mathrm{~Hz}) ;{ }^{13} \mathrm{C} \mathrm{NMR}\left(\mathrm{CDCl}_{3}, 75 \mathrm{MHz}\right) \delta 112.55$, 120.79 , 123.35, 125.65, 126.31, 127.32, 127.75, 128.99, 129.18, 129.89, 131.25, 132.00, 132.26, 134.98, 139.61, 139.75, 147.81, 150.61; Anal. Calcd. for $\mathrm{C}_{37} \mathrm{H}_{24} \mathrm{BrN}_{5}$ : C, 71.85; H, 3.91; N,11.32; found: C, 72.01; H, 4.06; N, 11.49 .

3,5-Bis-(4-tert-butylphenyl)-4-(2-chlorophenyl)-1,7-diphenyl-1,7-dihydrodipyrazolo[3,4$\boldsymbol{b} ; \mathbf{4}^{\prime}, \mathbf{3}^{\prime}-\boldsymbol{e}$ ]pyridine (10b). Yield $976 \mathrm{mg}(34 \%)$; light yellow solid; $\mathrm{mp}=274-277{ }^{\circ} \mathrm{C} ; \mathrm{R}_{\mathrm{f}}=0.48$ (toluene/PE, 1:1); ${ }^{1} \mathrm{H} \mathrm{NMR}\left(\mathrm{CDCl}_{3}, 300 \mathrm{MHz}\right) \delta 1.24(\mathrm{~s}, 18 \mathrm{H}, t-\mathrm{Bu}), 6.71(\mathrm{td}, 1 \mathrm{H}, J=7.5,1.5$ $\mathrm{Hz}), 6.82(\mathrm{td}, 2 \mathrm{H}, J=9.0,1.5 \mathrm{~Hz}), 6.93(\mathrm{ddd}, 1 \mathrm{H}, J=9.0,7.2,1.8 \mathrm{~Hz}), 7.02(\mathrm{~d}, 4 \mathrm{H}, J=8.7 \mathrm{~Hz})$, $7.07(\mathrm{~d}, 4 \mathrm{H}, J=8.7 \mathrm{~Hz}), 7.33(\mathrm{t}, 2 \mathrm{H}, J=7.5 \mathrm{~Hz}), 7.57(\mathrm{t}, 4 \mathrm{H}, J=7.5 \mathrm{~Hz}), 8.56(\mathrm{~d}, 4 \mathrm{H}, J=8.4$ $\mathrm{Hz}) ;{ }^{13} \mathrm{C} \mathrm{NMR}\left(\mathrm{CDCl}_{3}, 75 \mathrm{MHz}\right) \delta 31.17,34.42,112.85,120.69,124.21,125.49,128.58,128.76$, 128.96, 129.14, 129.35, 131.19, 133.00, 133.23, 138.30, 139.74, 147.94, 150.61, 150.67; Anal. Calcd. for $\mathrm{C}_{45} \mathrm{H}_{40} \mathrm{ClN}_{5}$ : C, 78.76; H, 5.87; N, 10.20; found: C, 78.60; H, 6.04; N, 10.01 .

4-(2-Bromophenyl)-3,5-bis-(4-tert-butylphenyl)-1,7-diphenyl-1,7-dihydrodipyrazolo[3,4-b; $\mathbf{4}^{\prime}, 3^{\prime}-\boldsymbol{e}$ ]pyridine (11b). Yield $2.16 \mathrm{~g}(29 \%)$; light yellow solid; $\mathrm{mp}=270-272{ }^{\circ} \mathrm{C} ; \mathrm{R}_{\mathrm{f}}=0.62$ (toluene); ${ }^{1} \mathrm{H} \mathrm{NMR}\left(\mathrm{CDCl}_{3}, 300 \mathrm{MHz}\right) \delta 1.23(\mathrm{~s}, 18 \mathrm{H}, t-\mathrm{Bu}), 6.74-6.87(\mathrm{~m}, 3 \mathrm{H}), 7.00-7.04(\mathrm{~m}$, $5 \mathrm{H}), 7.06(\mathrm{~d}, 4 \mathrm{H}, J=8.7 \mathrm{~Hz}), 7.33(\mathrm{t}, 2 \mathrm{H}, J=7.5 \mathrm{~Hz}), 7.57(\mathrm{t}, 4 \mathrm{H}, J=7.5 \mathrm{~Hz}), 8.57(\mathrm{~d}, 4 \mathrm{H}, J=$ $9.0 \mathrm{~Hz}) ;{ }^{13} \mathrm{C} \mathrm{NMR}\left(\mathrm{CDCl}_{3}, 75 \mathrm{MHz}\right) \delta 31.16,34.41,112.70,120.64,123.27,123.35,124.17$, $125.47,126.04,128.85,128.95,129.12$, 129.36, 131.23, 131.74, 134.91, 139.73, 139.80, 147.93, 150.62; Anal. Calcd. for $\mathrm{C}_{45} \mathrm{H}_{40} \mathrm{BrN}_{5}$ : C, 73.96; H, 5.52; N, 9.58; found: C, 73.61; H, 5.72; N, 9.69 .

4-(2-Chlorophenyl)-3,5-bis-(4-methoxyphenyl)-1,7-diphenyl-1,7-dihydrodipyrazolo[3,4-b; $\mathbf{4}^{\prime}, \mathbf{3}^{\prime}$-e $]$ pyridine (12c). Yield $1.48 \mathrm{~g}(21 \%)$; deep yellow solid; $\mathrm{mp}=247-249{ }^{\circ} \mathrm{C} ; \mathrm{R}_{\mathrm{f}}=0.24$ $\left(\mathrm{CHCl}_{3}\right) ;{ }^{1} \mathrm{H} \mathrm{NMR}\left(\mathrm{CDCl}_{3}, 300 \mathrm{MHz}\right) \delta 3.73(\mathrm{~s}, 6 \mathrm{H}, \mathrm{OMe}), 6.55(\mathrm{~d}, 4 \mathrm{H}, J=9.0 \mathrm{~Hz}), 6.82-6.91$ $(\mathrm{m}, 2 \mathrm{H}), 6.97-7.09(\mathrm{~m}, 6 \mathrm{H}), 7.33(\mathrm{t}, 2 \mathrm{H}, J=7.5 \mathrm{~Hz}), 7.56(\mathrm{t}, 4 \mathrm{H}, J=7.5 \mathrm{~Hz}), 8.53(\mathrm{~d}, 4 \mathrm{H}, J=$ $9.0 \mathrm{~Hz}) ;{ }^{13} \mathrm{C} \mathrm{NMR}\left(\mathrm{CDCl}_{3}, 75 \mathrm{MHz}\right) \delta 55.23,112.60,112.92,114.53,120.71,124.77,125.50$, $125.84,128.94,129.76,130.26,131.29,133.19,133.29,138.22,139.64,147.56,150.56$, 159.264; Anal. Calcd. for $\mathrm{C}_{39} \mathrm{H}_{28} \mathrm{ClN}_{5} \mathrm{O}_{2}$ : C, 73.87; H, 4.45; N, 11.04; found: C, 74.07; H, 4.62; N, 10.91.

\section{General procedure for the preparation of the TPPACIs $(\operatorname{method} \mathrm{A})$}

9a $(500 \mathrm{mg}, 0.808 \mathrm{mmol})$, powdered $\mathrm{KOH}(1.5 \mathrm{~g}, 27 \mathrm{mmol})$ and isoquinoline $(10 \mathrm{~mL})$ were heated at reflux for 1 hour. After cooling water $(50 \mathrm{~mL})$ was added. The mixture was extracted with toluene ( 2 x $50 \mathrm{~mL})$. Organic phase was washed with $4 \mathrm{M} \mathrm{HCl}(2 \times 25 \mathrm{~mL})$ and finally with saturated aqueous $\mathrm{NaCl}$ solution. After drying $\left(\mathrm{Na}_{2} \mathrm{SO}_{4}\right)$ the solvent was removed to dryness and the yellow residue was dissolved in $\mathrm{CHCl}_{3}$. The product was precipitated by a slow addition of $\mathrm{MeOH}$. For 8a the same amounts of reagents were used, however the mixture was heated at reflux for 2 hours.

3,5-Dihydro-1,3,5-triphenyl-2,3,4,5,6-pentaazadibenzo[4,5:6,7]cyclohepta[1,2,3-cd]s-indacene (14a). Yield $430 \mathrm{mg}(99 \%)$ from 9a and $390 \mathrm{mg}(83 \%)$ from 8a; yellow solid; $\mathrm{mp}=282-284{ }^{\circ} \mathrm{C}$; $\mathrm{R}_{\mathrm{f}}=0.47$ (toluene/PE); (note: about $7 \mathrm{mg}$ of $\mathbf{1 4 a}$ can be dissolved in $0.87 \mathrm{~mL}$ of $\mathrm{CDCl}_{3}$ at room 
temperature); ${ }^{1} \mathrm{H}$ NMR $\left(\mathrm{CDCl}_{3}, 300 \mathrm{MHz}\right) \delta ;{ }^{13} \mathrm{C} \mathrm{NMR}\left(\mathrm{CDCl}_{3}, 75 \mathrm{MHz}\right) \delta 6.83$ (ddd, $1 \mathrm{H}, J=$ 8.1, 7.2, $1.2 \mathrm{~Hz}), 7.25(\mathrm{dd}, 1 \mathrm{H}, J=8.1,1.2 \mathrm{~Hz}), 7.28-7.38(\mathrm{~m}, 6 \mathrm{H}), 7.50-7.61(\mathrm{~m}, 6 \mathrm{H})$, 7.65-7.71 (m, 3H), 7.88-7.94 (m, 1H), 8.38-8.45 (m, 1H), $8.52(\mathrm{~d}, 2 \mathrm{H}, J=8.7 \mathrm{~Hz}), 8.56(\mathrm{~d}, 2 \mathrm{H}$, $J=8.7 \mathrm{~Hz}$ ); Anal. Calcd. for $\mathrm{C}_{37} \mathrm{H}_{23} \mathrm{~N}_{5}: \mathrm{C}, 82.66 ; \mathrm{H}, 4.31 ; \mathrm{N}, 13.03$; found: C, 82.35; H, 4.41; N, 13.03 .

9-(tert-Butyl)-1-[4-(tert-butyl)phenyl]-3,5-dihydro-3,5-diphenyl-2,3,4,5,6-pentaazadibenzo [4,5:6,7]cyclohepta[1,2,3-cd]s-indacene (14b). Yield $227 \mathrm{mg}(51 \%)$ from $11 \mathrm{~b}$ and $284 \mathrm{mg}$ $(60 \%)$ from 10b; deep yellow solid after column purification; $\mathrm{mp}=320-322{ }^{\circ} \mathrm{C} ; \mathrm{R}_{\mathrm{f}}=0.82$ (toluene); ${ }^{1} \mathrm{H} \mathrm{NMR}\left(\mathrm{CDCl}_{3}, 300 \mathrm{MHz}\right) \delta 1.31(\mathrm{~s}, 9 \mathrm{H}, t$-Bu), $1.46(\mathrm{~s}, 9 \mathrm{H}, t$-Bu), 6.72 (ddd, $1 \mathrm{H}, J$ $=7.9,7.2,1.2 \mathrm{~Hz}), 7.16(\mathrm{dd}, 1 \mathrm{H}, J=8.1,1.2 \mathrm{~Hz}), 7.23-7.34(\mathrm{~m}, 5 \mathrm{H}), 7.51-7.59(\mathrm{~m}, 7 \mathrm{H}), 7.62$ $(\mathrm{dd}, 1 \mathrm{H}, J=8.1,1.2 \mathrm{~Hz}), 7.87(\mathrm{~d}, 1 \mathrm{H}, J=2.1 \mathrm{~Hz}), 8.32(\mathrm{~d}, 1 \mathrm{H}, J=8.1 \mathrm{~Hz}), 8.52(\mathrm{~d}, 2 \mathrm{H}, J=8.7$ $\mathrm{Hz}), 8.53(\mathrm{~d}, 2 \mathrm{H}, J=8.7 \mathrm{~Hz}) ;{ }^{13} \mathrm{C} \mathrm{NMR}\left(\mathrm{CDCl}_{3}, 75 \mathrm{MHz}\right) \delta 31.31,31.32,34.66,34.96,109.21$, $119.47,120.24$, 121.08, 124.98, 125.25, 125.56, 126.08, 126.33, 127.35, 128.88, 128.90, 128.94, $129.10,129.57,130.47,130.54,132.74,134.15,136.03,137.45,139.77,139.94,140.32,140.74$, 144.48, 147.01, 150.55, 151.45, 151.88, 152.93; Anal. Calcd. for $\mathrm{C}_{45} \mathrm{H}_{39} \mathrm{~N}_{5}$ : C, 83.17; H, 6.05; N, 10.78; found: C, 82.98; $\mathrm{H}, 6.00 ; \mathrm{N}, 10.65$.

3,5-Dihydro-9-methoxy-1-(4-methoxyphenyl)-3,5-diphenyl-2,3,4,5,6-pentaazadibenzo [4,5:6,7]cyclohepta[1,2,3-cd]s-indacene (14c). Yield $403 \mathrm{mg}(85 \%)$ from 12c; yellow solid, no column required; $\mathrm{mp}=291-293{ }^{\circ} \mathrm{C} ; \mathrm{R}_{\mathrm{f}}=0.39\left(\mathrm{CHCl}_{3}\right) ;$ (note: about $5 \mathrm{mg}$ of $14 \mathrm{c}$ can be dissolved in $0.87 \mathrm{~mL}$ of $\mathrm{CDCl}_{3}$ at room temperature); ${ }^{1} \mathrm{H} \mathrm{NMR}\left(\mathrm{CDCl}_{3}, 300 \mathrm{MHz}\right) \delta 3.82(\mathrm{~s}, 3 \mathrm{H}$, OMe), 3.98 (s, 3H, OMe), 6.84 (d, 2H, $J=9.0 \mathrm{~Hz}$ ), 6.89 (ddd, 1H, J=8.1,7.2, 1.2 Hz), 7.12 (dd, $1 \mathrm{H}, J=8.7,2.7 \mathrm{~Hz}), 7.28-7.37(\mathrm{~m}, 4 \mathrm{H}), 7.42(\mathrm{~d}, 1 \mathrm{H}, J=2.7 \mathrm{~Hz}), 7.54-7.63(\mathrm{~m}, 6 \mathrm{H}), 7.74(\mathrm{dd}$, $1 \mathrm{H}, J=8.1,1.2 \mathrm{~Hz}), 8.35(\mathrm{~d}, 1 \mathrm{H}, J=8.7 \mathrm{~Hz}), 8.52(\mathrm{~d}, 2 \mathrm{H}, J=8.7 \mathrm{~Hz}), 8.56(\mathrm{~d}, 2 \mathrm{H}, J=8.7 \mathrm{~Hz})$; Anal. Calcd. for $\mathrm{C}_{39} \mathrm{H}_{27} \mathrm{~N}_{5} \mathrm{O}_{2}$ : C, 78.38; H, 4.55; N, 11.72; found: C, 78.15; H, 4.68; N, 11.68 .

\section{Palladium-assisted cyclization of (9a) and (11b) (method B)}

9a (100 mg, $0.162 \mathrm{mmol}), \mathrm{Pd}_{2}(\mathrm{dba})_{3}(30 \mathrm{mg}, 0.032 \mathrm{mmol}, 20 \% \mathrm{~mol})$ and DBU $(0.25 \mathrm{~mL})$ were dissolved in dry DMF $(10 \mathrm{~mL})$. The mixture was purged with argon for $10 \mathrm{~min}$. Next $\mathrm{P}(t-\mathrm{Bu})_{3}$ $\left(0.13 \mathrm{~mL}, 1 \mathrm{M}\right.$ in toluene) was added in one portion, which was heated at $130{ }^{\circ} \mathrm{C}$ for 5 hours. After cooling, water was added and the yellow solid that precipitated was extracted with toluene. After drying and concentration, the oily residue was passed through a short column packed with alumina. 14a, yield: $80 \mathrm{mg}(92 \%)$. For 14b: $11 b(150 \mathrm{mg}, 0.205 \mathrm{mmol}), \mathrm{Pd}_{2}(\mathrm{dba})_{3}(37.5 \mathrm{mg}$, $0.041 \mathrm{mmol}, 20 \% \mathrm{~mol}), \mathrm{DBU}(0.25 \mathrm{~mL}), \mathrm{P}(t-\mathrm{Bu})_{3}(0.16 \mathrm{~mL}, 1 \mathrm{M}$ in toluene $)$ and $\mathrm{DMF}(10 \mathrm{~mL})$. Yield $98 \mathrm{mg}(74 \%)$.

\section{Preparation of doubly annulated aza s-indacenes}

The precursors 16a and 16b were prepared as for 8-12.

3,5-Bis(2-chlorophenyl)-1,4,7-triphenyl-1,7-dihydrodipyrazolo[3,4-b:4',3'-e]pyridine (16a). Yield $1.18 \mathrm{~g}(26 \%)$; colorless solid; $\mathrm{mp}=264-266{ }^{\circ} \mathrm{C} ; \mathrm{R}_{\mathrm{f}}=0.59$ (toluene); ${ }^{1} \mathrm{H} \mathrm{NMR}\left(\mathrm{CDCl}_{3}\right.$, $300 \mathrm{MHz}) \delta 6.64(\mathrm{t}, 2 \mathrm{H}, J=7.2 \mathrm{~Hz}), 6.83-6.89(\mathrm{~m}, 3 \mathrm{H}), 7.02-7.13(\mathrm{~m}, 6 \mathrm{H}), 7.19-7.24(\mathrm{~m}, 2 \mathrm{H})$, 
$7.34(\mathrm{t}, 2 \mathrm{H}, J=7.5 \mathrm{~Hz}), 7.58(\mathrm{t}, 4 \mathrm{H}, J=7.5 \mathrm{~Hz}), 8.53(\mathrm{~d}, 4 \mathrm{H}, J=8.7 \mathrm{~Hz}) ;{ }^{13} \mathrm{C} \mathrm{NMR}\left(\mathrm{CDCl}_{3}, 75\right.$ $\mathrm{MHz}) \delta 113.37,120.90,125.72,125.95,126.02,127.72,128.89,128.99,129.54,129.62,131.03$, 131.64, 132.40, 134.15, 139.58, 142.50, 145.02, 150.42; Anal. Calcd. for $\mathrm{C}_{37} \mathrm{H}_{23} \mathrm{Cl}_{2} \mathrm{~N}_{5}$ : C, 73.03; H, 3.81; N, 11.51; found: C, 72.95; H, 3.91; N, 11.60.

4-(4-tert-Butylphenyl)-3,5-bis-(2-chlorophenyl)-1,7-diphenyl-1,7-dihydrodipyrazolo[3,4$\boldsymbol{b} ; \mathbf{4}^{\prime}, \mathbf{3}^{\prime}-\boldsymbol{e}$ ]pyridine (16b). Yield $1.84 \mathrm{~g}(28 \%)$; colorless solid; $\mathrm{mp}=297-300{ }^{\circ} \mathrm{C} ; \mathrm{R}_{\mathrm{f}}=0.59$ (toluene); ${ }^{1} \mathrm{H} \mathrm{NMR}\left(\mathrm{CDCl}_{3}, 300 \mathrm{MHz}\right) \delta 1.13(\mathrm{~s}, 9 \mathrm{H}, t-\mathrm{Bu}), 6.62(\mathrm{~d}, 2 \mathrm{H}, J=8.7 \mathrm{~Hz}), 6.70(\mathrm{~d}$, $2 \mathrm{H}, J=8.1 \mathrm{~Hz}), 6.97-7.08(\mathrm{~m}, 6 \mathrm{H}), 7.16(\mathrm{dd}, 2 \mathrm{H}, J=6.6 \mathrm{~Hz}), 7.34(\mathrm{t}, 2 \mathrm{H}, J=7.2 \mathrm{~Hz}), 7.58(\mathrm{t}$, $4 \mathrm{H}, J=8.7 \mathrm{~Hz}), 8.54(\mathrm{~d}, 4 \mathrm{H}, J=8.7 \mathrm{~Hz}) ;{ }^{13} \mathrm{C} \mathrm{NMR}\left(\mathrm{CDCl}_{3}, 75 \mathrm{MHz}\right) \delta 31.01,34.19,113.48$, $120.85,122.76,125.65,125.89$, 127.95, 128.80, 128.97, 129.12, 129.38, 131.70, 132.39, 134.16, 139.64, 142.83, 145.12, 150.44, 150.51; Anal. Calcd. for $\mathrm{C}_{41} \mathrm{H}_{31} \mathrm{Cl}_{2} \mathrm{~N}_{5}: \mathrm{C}, 74.09 ; \mathrm{H}, 4.70 ; \mathrm{N}$, 10.54; found: C, 74.21; H, 4.65; N, 10.50 .

\section{1-tert-Butyl-2,4-diphenyl-2,4-dihydro-1,2,3,4,5-pentaazatribenzo[3,4:5,6:7,8]heptaleno}

[2,1,10,9-cdef]s-indacene (17b). 16b (500 mg, $0.753 \mathrm{mmol}), \mathrm{KOH}(2.53 \mathrm{~g}, 45 \mathrm{mmol})$ and isoquinoline $(10 \mathrm{~mL})$ were heated at reflux for 2 hours. After cooling, water $(50 \mathrm{~mL})$ was added, followed by toluene $(50 \mathrm{~mL})$. The inorganic phase was discarded, and isoquinoline was extracted with $4 \mathrm{M} \mathrm{HCl}$. The organic phase was washed with saturated aqueous $\mathrm{NaCl}(50 \mathrm{~mL})$ and dried $\left(\mathrm{K}_{2} \mathrm{CO}_{3}\right)$. After evaporation, the solid residue was dissolved in toluene and passed through a short column packed with alumina. After evaporation of the volatiles the red crystals were dissolved in chloroform and $\mathrm{MeOH}$ was added dropwise with stirring. The red amorphous solid was filtered off. Yield $359 \mathrm{mg}(81 \%) ; \mathrm{R}_{\mathrm{f}}=0.76$ (toluene); $\mathrm{mp}=339-341{ }^{\circ} \mathrm{C} ;{ }^{1} \mathrm{H} \mathrm{NMR}\left(\mathrm{CDCl}_{3}\right.$, $300 \mathrm{MHz}) \delta 1.41(\mathrm{~s}, 9 \mathrm{H}, t-\mathrm{Bu}), 7.20-7.23(\mathrm{~m}, 2 \mathrm{H}), 7.30(\mathrm{t}, 2 \mathrm{H}, J=7.2 \mathrm{~Hz}), 7.42-7.49(\mathrm{~m}, 4 \mathrm{H})$, $7.53(\mathrm{t}, 4 \mathrm{H}, J=7.2 \mathrm{~Hz}), 7.55(\mathrm{~s}, 2 \mathrm{H}), 8.31-8.34(\mathrm{~m}, 2 \mathrm{H}), 8.45(\mathrm{~d}, 4 \mathrm{H}, J=8.7 \mathrm{~Hz}) ;{ }^{13} \mathrm{C} \mathrm{NMR}$ $\left(\mathrm{CDCl}_{3}, 75 \mathrm{MHz}\right) \delta 30.94,34.76,113.21,120.76,125.48,126.21,128.59,128.88,129.10$, 130.94, 131.03, 132.85, 134.44, 137.33, 139.71, 140.07, 142.43, 144.84, 152.44, 153.26; Anal. Calcd. for $\mathrm{C}_{41} \mathrm{H}_{29} \mathrm{~N}_{5}$ : C, 83.22; H, 4.94; N, 11.84; found: C, 83.42; H, 4.83; N, 11.80 .

\section{Acknowledgements}

Dr. hab. Andrzej Danel is kindly acknowledged for his donation of 1,3-diphenyl-1H-pyrazol-5amine 7a.

\section{References}

1. (a) Danel, K. S.; Wisła, A.; Uchacz, T. Arkivoc 2009, (x), 71. (b) Całus, S.; Danel, K. S.; Uchacz, T.; Kityk, A. V. Mater. Chem. Phys. 2010, 121, 477. (c) Danel, K. S; Gąsiorski, P.; Matusiewicz, M.; Całus, S.; Uchacz, T.; Kityk, A. V. Spectrochim. Acta A 2010, 77, 16. (d) Gąsiorski, P.; Danel, K. S.; Matusiewicz, M.; Uchacz, T.; Kityk, A. V. J. Lumin. 2010, 130, 
2460. (e) Gąsiorski, P.; Danel, K. S.; Matusiewicz, M.; Uchacz, T.; Vlokh, R.; Kityk, A. V. J. Fluoresc. 2011, 21, 443.

2. (a) Kaoudi, T.; Quiclet-Sire, B.; Seguin, S.; Zard, S. Z. Angew. Chem. Int. Ed. 2000, 39, 731.

(b) Hirama, Y.; Abe, H.; Minakawa, N.; Matsuda, A. Tetrahedron 2010, 66, 8402.

3. (a) Crespi, V. H.; Cohen, M. L.; Louie, S. G. S.; Zettl, A. K. U. S. Patent 6,835,952, 2004; Chem. Abstr. 2003, 138, 264103. (b) Ponomareva, I.; Chernozatonskii, L. A.; Andriotis, A. N.; Menon, M. New J. Phys. 2003, 5, 119.1.

4. Matschke, M.; Beckert, R.; Würthwein, E.-U.; Görls, H. Synlett 2008, 17, 2633.

5. Katz, A. K.; Carrell, H. L.; Glusker, J. P. Carcinogenesis 1998, 19, 1641.

6. Brulé, C.; Laali, K. K.; Okazaki, T.; Lakshman, M. K. J. Org. Chem. 2007, 72, 3232.

7. Sahasithiwat, S.; Mophuang, T.; Menbangpung, L.; Kamtonwong, S.; Sooksimuang, T. Synth. Met. 2010, 160, 1148.

8. (a) He, Z.; Milburn, G. H. W.; Danel, A.; Puchała, A.; Tomasik, P.; Rasała, D. J. Mater. Chem. 1997, 7, 2323. (b) Balasubramaniam, E.; Tao, Y.T.; Danel, A.; Tomasik, P. Chem. Mater. 2000, 12, 2788. (c) Tao, Y. T.; Balasubramaniam, E.; Danel, A.; Tomasik, P. Appl. Phys. Lett. 2000, 77, 933. (d) Tao, Y. T.; Chuen, C. H.; Ko, C. W.; Peng, J. W. Chem. Mater. 2002, 14, 4256.

9. Huang, T.-H.; Lin, J.T.; Tao, Y.-T.; Chuen, C.-H. Chem. Mat. 2003, 15, 4854.

10. (a) Smet, M.; Dehaen, M. Molecules 2000, 5, 620. (b) De Backer, S.; Prinzie, J.; Verheijen, W.; Smet, M.; Desmedt, K.; Dehaen, W.; De Schryver, F. C. J. Phys. Chem. 1998, 102, 5451.

11. (a) Drauz, K. H.; Kleemann, A.; Wolf-Heuss, E. Ger. Offen. DE 3209 472, 1983; Chem. Abstr. 1983, 100, 51289. (b) Baraldi,P. G.; Romagnoli, R.; Pavani, M. G.; Nuñez, M. C.; Tabrizi, M. A.; Shryock, J. C.; Leung, E.; Moorman, A. R.; Uluoglu, C.; Iannotta, V.; Merighi,, S.; Borea, P. A. J. Med. Chem. 2003, 46, 794.

12. (a) Wang, P.; Xie, Z.; Hong, Z.; Tang, J.; Wong, O.; Lee, C.-S.; Wong, N.; Lee, S. J. Mater. Chem. 2003, 13, 1894. (b) 7a-c: Reddy, G. J.; Latha, D.; Rao, K. S. Org. Prep. Proced. Int. 2004, 36, 494. (c) 15: Moe, S. T.; Thompson, A. B.; Smith, G. M.; Fredenburg, R. A.; Stein, R. L.; Jacobson, A. R. Bioorg. Med. Chem. 2009, 17, 3072.

13. Hennig, L.; Hofmann, J.; Alva-Astudillo, M.; Mann, G. J. Prakt. Chem. 1990, 332, 351. 